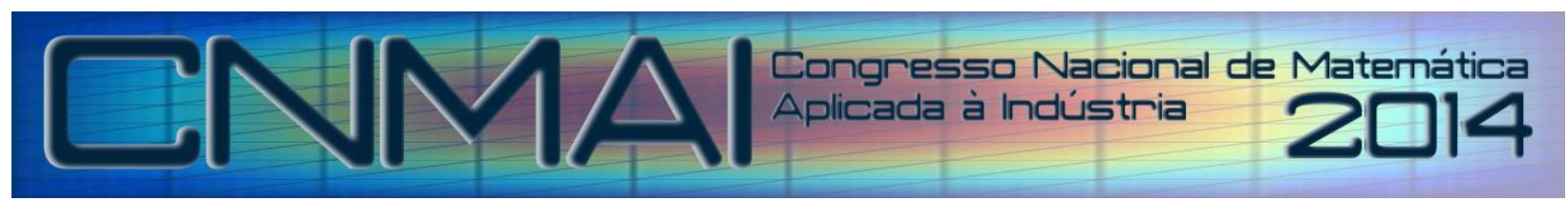

18 a 21 de novembro de 2014, Caldas Novas - Goiás

\title{
AVANÇOS NA MODELAGEM COM O METODO DE VOLUMES FINITOS DE UMA CALDEIRA PILOTO DE BIOMASSA EM LEITO FIXO.
}

\author{
Juan Carlos Guerrero Ordoñez, juank2585@ hotmail.com ${ }^{1}$ \\ ${ }^{1}$ Universidade Federal de Itajubá - Enghenaria de Energia - NEST. Rua Dr. Pereira Cabral, 1303, Pinheirinho, Itajubá - \\ MG, 37500-903, Brasil.
}

\begin{abstract}
Resumo: O incremento do consumo energético mundial e os padrões permitidos de poluentes emitidos na atmosfera, além de incrementar a eficiência nos processos de conversão da energia (química-mecânica-elétrica) são as principais razões da investigação no âmbito de engenharia e energia na atualidade. A geração de energia elétrica a partir da queima de biomassa em caldeiras pequenas faz parte deste grupo de pesquisas. A caldeira piloto do estudo é aquatubular de $60 \mathrm{~kg} / \mathrm{h}$ de produção de vapor e $90 \mathrm{kWT}$ de capacidade térmica. A biomassa utilizada foi pedaços de madeira que são queimados em camada sobre a grelha. A transformação das propriedades físico-químicas na caldeira depende do processo de combustão na fornalha, assim as equações empíricas da combustão estão relacionadas com: reações químicas, transferência de calor, radiação e dinâmica de fluidos. Sua complexidade matemática, a dificuldade de testar, controlar as variáveis do processo e a diminuição de custos faz da modelagem uma ferramenta de grande interesse pela engenharia. A metodologia utilizada neste artigo sobre a modelagem das caldeiras com queima em grelha fixa permite avaliar propriedades para diminuir perdas, melhorar o projeto inicial e controlar a formação de NOx e CO. A metodologia é baseada em estudos semelhantes e considerações próprias. Os avanços da modelagem mostrados neste artigo foram feitos com um software comercial. Os perfis de velocidade, pressão e temperaturas dentro da caldeira são calculados.
\end{abstract}

Palavras-chave: Modelagem matematico, Volumens finitos, Caldeira, Biomassa, CFD.

\section{INTRODUÇÃO}

Historicamente a energia foi requerida para abastecer as principais necessidades humanas um exemplo específico é o fornecimento do calor na preparação da comida e na fabricação de ferramentas para caça, entre muitas outras aplicações, posteriormente devido à revolução industrial o consumo da energia aumento exponencialmente, desse modo a humanidade começou a sofrer problemas ambientais como o aquecimento global: o aumento da temperatura, os fenômenos como furacões e tornados e a morte de algumas espécies da flora e fauna são consequência desta atividade.

No momento atual a engenharia é uma ferramenta utilizável para gerir a oferta e demanda energética nos países. Também é aplicada para diminuir os poluentes principalmente fornecidos pela indústria, um claro exemplo são os numerosos projetos de eficiência energética e do uso de biocombustíveis "CO2 neutral" na produção de energia. O aproveitamento energético dos recursos e os resíduos agrícolas são considerados de muita importância para lograr a diminuição do uso das fontes fosseis e do $\mathrm{CO}_{2}$.

Devido à diversidade de recursos de biomassa, suas aplicações não estão limitadas à produção de combustíveis ou de eletricidade, produtos auxiliares também podem ser produzidos durante o processo de conversão como usando três principais tecnologias de conversão; bioquímica, termoquímica e processos de extração. Alguns processos termoquímicos de conversão de energia utilizável a partir da biomassa são: Combustão, gasificação, pirolises, digestão, fermentação e extração.

O grupo de pesquisa NEST (Núcleo de Excelência em Geração Termelétrica e Distribuída) da Universidade Federal de Itajubá trabalha em projetos de geração de energia elétrica a partir das fontes renováveis, em sua abordagem encontra-se a optimização de sistemas geradores de vapor. Neste trabalho se descrevem alguns avanços da metodologia para a modelagem com o método de Volumes Finitos de uma caldeira piloto com o objetivo de avaliar o processo de combustão, otimizar o projeto da caldeira e reduzir a formação de poluentes.

\section{FUNDAMENTOS TEÓRICOS}

O engenheiro ou projetista incumbindo de resolver um determinado problema tem a sua disposição fundamentalmente três ferramentas: 1. Métodos analíticos, 2. Métodos numéricos, 3. Experimentação em laboratório. A experimentação numérica, presenta poucas restrições, podendo resolver problemas com complicadas condições de contorno definidos em geometrias arbitrárias e apresentado resultados com maior rapidez, se os recursos 
computacionais estiverem disponíveis. O método de Volumes Finitos faz parte de este grupo. O analises feito neste trabalho foi realizado com a Dinâmica de Fluidos Computacional (CFD) com o software comercial Ansys Fluent ${ }^{\circledR}$, o CFD usa o método de Volumes Finitos para a solução das equações da Dinâmica de Fluidos, incluindo transferência de calor, radiação e fontes térmicas.

\subsection{Método de Volumes Finitos}

O método de volumes finitos (MVF) utiliza como ponto de partida a forma integral da equação da conservação. O domínio de solução é dividido num número finito de volumes de controle (VC) contíguos, e a equação da conservação é aplicada a cada VC. No centroide de cada VC localiza-se um nó computacional, no qual são calculados os valores das variáveis, sendo os valores das variáveis nas superfícies dos VC obtidos por interpolação em função dos valores nodais (centro do VC). Os integrais de volume e de superfície são aproximados usando fórmulas de quadratura apropriadas. Como resultado, obtém-se uma equação algébrica para cada VC, na qual aparecem os valores das variáveis no nó em causa e nos nós vizinhos.

\subsection{Dinâmica de Fluidos Computacional}

As equações que governam os escoamentos viscosos incompressíveis com propriedades constantes e desprezando as forças do corpo causadas por um campo gravitacional são: equação de conservação de massa (continuidade), equação de conservação da quantidade de movimento e a equação da conservação da energia.

Na figura 1. Mostra-se a relação das equações que governam a dinâmica de fluidos com os princípios fundamentais da física.

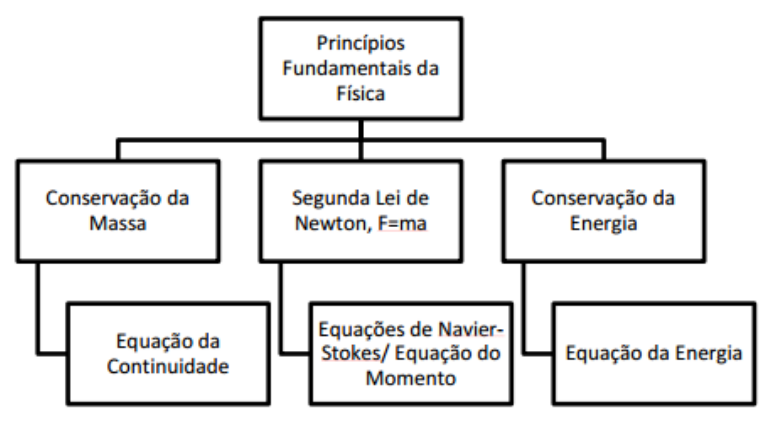

Figura 1. Relações entre os princípios fundamentais da Física e as equações que governam a dinâmica dos Fluidos.

A equação de Navier Stokes é tratada no analises dos escoamentos com regímen turbulento devido a que sua a aplicação é relacionada com este caso especifico.

\subsection{Escoamentos em Regímen turbulento}

Segundo Reynolds, a velocidade instantânea num escoamento com regímen turbulento pode ser decomposta em duas componentes, um componente médio e um componente de flutuação.

Desta forma, a velocidade instantânea segundo a direção i vem dada por:

$$
\nu_{i}=\bar{\nu}_{i}+v_{i}
$$

Em que $\boldsymbol{v}_{i}$ corresponde à velocidade instantânea, $\bar{\nu}_{i}$ à velocidade média e $\boldsymbol{v}_{i}^{*}$ a velocidade flutuante.

\subsection{Lei da parede}

Experimentalmente se demostrou que em escoamentos turbulentos o perfil da velocidade na camada limite, consiste basicamente de três camadas, uma interna, outra externa e mais uma intermedia, na qual se interpõem as duas anteriores. Figura 3. 


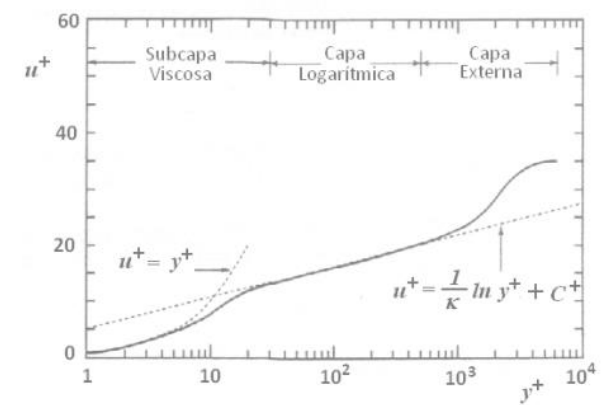

Figura 2. Perfil de velocidades na camada limite no escoamento perto das paredes usando variáveis adimensionais $\mathbf{y}^{+} \mathbf{e} \mathbf{u}^{+}$.

Na região perto das paredes a camada interna é conhecida como subcamada viscosa, nesta a transferência de momentum se dá principalmente devido ao efeito de viscosidade molecular, o seja, os esforços cortantes viscosos dominam esta região, tanto que na camada externa (região completamente turbulenta) os esforços de Reynolds são dominantes. Na camada intermedia tanto os esforços viscosos como os esforços de Reynolds são importantes.

A equação da lei da parede é:

$$
\frac{\bar{u}}{u_{\tau}}=\frac{1}{k} \ln \left(\frac{y u_{\tau}}{v}\right)+C^{+}
$$

Onde $u_{\tau}$ é a velocidade de fricção, $\mathrm{k}$ é conhecida como a constante de Kárman e é aproximadamente $0,41, C^{+}$é uma constante adimensional igual a 5 para paredes lisas.

A lei da parede tem variáveis adimensionais de velocidade e distancia:

$$
u^{+}=\frac{\bar{u}}{u_{\tau}} \quad y^{+}=\frac{y u_{\tau}}{v}
$$

Os anteriores termos adimensionais da lei da parede definiram o lugar do analises na fornalha da caldeira e são calculados no capitulo $5, \mathrm{o} \mathrm{y}^{+}$tem relação direita com a projeção da malha da modelagem.

\subsection{Equação de Reynolds}

As equações de Navier Stokes medias temporalmente conhecem-se como RANS são tratadas em escoamentos turbulentos, para escoamentos incompreensíveis se podem escrever assim:

Equação de continuidade:

$$
\frac{\partial \bar{u}_{i}}{\partial x_{i}}=0
$$

Equação de momento:

$$
\begin{array}{r}
\frac{\partial}{\partial t}\left(\rho \bar{u}_{i}\right)+\frac{\partial}{\partial x_{i}}\left(\rho \bar{u}_{i} \bar{u}_{j}\right)=-\frac{\partial \bar{p}}{\partial x_{i}}+\frac{\partial}{\partial x_{i}}\left(2 \mu S_{i j}-\rho \overline{u_{i}^{\prime} u_{j}^{\prime}}\right) \\
\left(\bar{\tau}_{i j}\right)_{L a m}=2 \mu S_{i j}, \quad\left(\bar{\tau}_{i j}\right)_{T u r b}=-\rho \overline{u_{i}^{\prime} u_{j}^{\prime}}
\end{array}
$$

Onde $\left(\bar{\tau}_{i j}\right)_{\text {Lam é o esforço cortante laminar, }} S_{i j}$ é a taxa média de deformação e $\left({ }^{\bar{\tau}_{i j}}\right)$ Turbulento é um esforço cortante conhecido como Esforço de Reynolds.

O Esforço de Reynolds é a maior problema para resolver as equações RANS devido que as novas variáveis introduzidas neste termo não são conhecidas. 


\subsection{Modelos de Turbulência}

Para poder resolver as equações RANS existem os modelos de turbulência, os quais se dividem em três grupos: Os de primeira categoria que usam a suposição de Boussnesq, A segunda categoria usa a suposição das equações de Reynolds e a terceira categoria são equações baseadas nas equações de Reynolds como é o modelo LES e DNS. Na tabela 1 descreve-se os modelos de turbulência da categoria 1 que são os analisados neste estudo.

Tabela 1. Desenvolvimento e aplicação dos modelos de turbulência de categoria 1.

\begin{tabular}{|l|l|}
\hline Modelo & Descrição \\
\hline k- $\varepsilon$ & $\begin{array}{l}\text { Modelo que utilizam duas equações de transporte, uma para a energia cinética turbulenta (k) e outra para a taxa } \\
\text { de dissipação da turbulência }(\varepsilon) \text {. }\end{array}$ \\
\hline RNG k- $\varepsilon$ & $\begin{array}{l}\text { Este modelo é um refinamento do modelo k- } \varepsilon \text {, sendo que é derivado diretamente a partir das equações de Navier- } \\
\text { Stokes instantâneas, usando uma técnica matemática denominada por "Renormalization Group Method" (RNG). }\end{array}$ \\
\hline Realizable k- $\varepsilon$ & $\begin{array}{l}\text { Este modelo é um desenvolvimento recente do modelo k- } \varepsilon \text { e difere deste em dois aspetos importantes: - } \\
\text { Apresenta uma nova formulação para } \mu \text { - É usada uma nova equação de transporte para } \varepsilon\end{array}$ \\
\hline k- $\omega$ & $\begin{array}{l}\text { Este modelo utiliza uma versão modificada da equação de transporte de usada no modelo k- } \varepsilon \text { e outra equação de } \\
\text { transporte para a taxa de dissipação específica ( } \omega)\end{array}$ \\
\hline SST k- $\omega$ & $\begin{array}{l}\text { O modelo "Shear Stress Transport" (SST) é uma variante do modelo k- } \omega . \text { Este é uma combinação dos modelos } \\
\text { padrão k- } \omega \text { e k- } \varepsilon \text {, sendo que o primeiro é aplicado para zonas próximas de parede e o segundo para as zonas mais } \\
\text { afastadas da parede. }\end{array}$ \\
\hline Transition SST & Este modelo é baseado no modelo SST k- $\omega$, sendo que são duas equações de transporte adicionais resolvidas. \\
\hline
\end{tabular}

\subsection{Descrição da modelagem do leito fixo como meio poroso.}

O modelo de meio poroso pode ser usado para escoamentos que passam através de: leitos, filtros de papel, placas perfuradas, distribuidores de fluxo e bancos de tubos, o escoamento pode estar em uma ou várias fases. No modelo de meio poroso a caída de pressão é determinada pela condição de entrada nas equações de momento para meio poroso e de transferência de calor as quais podem ser formuladas em equilíbrio térmico ou não, através da equação do tratamento da energia para meio poroso. $\mathrm{O}$ efeito da turbulência no meio é aproximado.

O meio poroso é modelado pela adição de um termo que representa uma fonte de momento nas equações padrão de governo. Esse termo fonte é composto por duas partes: um termo de perdas viscosas (Lei de Darcy, parte esquerda da esquerda equação 10), e o outro termo representa as perdas inerciais (parte direita da equação 10).

$$
S_{i}=-\left(\sum_{j=1}^{3} D_{i j} \mu v_{j}+\sum_{j=1}^{3} C_{i j} \frac{1}{2} \rho|v| v_{j}\right)
$$

Onde Si é o termo fonte na dimensão i (x, y ou z) da equação do momento, $|v|$ é o módulo da velocidade, $\mathrm{D}$ e C são matrizes. O dissipador do momento cria uma caída de pressão que é proporcional à velocidade superficial do fluido na região porosa. No caso do meio poroso homogêneo se tem que:

$$
S_{i}=-\left(\frac{\mu}{\alpha} v_{i}+C_{2} \frac{1}{2} \rho|v| v_{i}\right)
$$

Onde $\alpha$ é a permeabilidade e C2 é o fator da resistência inercial.

Os dois termos anteriores representam a resistência do leito fixo no escoamento e são introduzidos na caraterização da camada do combustível na modelagem. Para calcular a perda de pressão em um reator de leito de recheio poroso é usada a equação de Ergun:

$$
\frac{d P}{d Z}=-\frac{G}{\rho g_{c} D_{p}}\left(\frac{1-\Phi}{\Phi^{g}}\right)\left[\frac{150(1-\Phi) \mu}{D_{p}}+1,75 G\right]
$$

Onde, $\mathrm{P}=$ pressão, $\mathrm{lb} / \mathrm{ft} . \Phi=$ porosidade (volume de vazios/volume total do leito). $1-\Phi=$ (volume de solido/volume total do leito). $g_{c}=32,174 l b_{m} \times f t / s^{2} \times l b_{f}$ (Fator de conversão), $=4,17 \times 108 l b_{m} \times f t / s^{2} \times l b_{f}$, (lembrese que para o sistema métrico, $g_{c}=1$ ). Dp = Diâmetro da partícula no leito, ft. $\mu=$ viscosidade do gás passando através do leito, ft. $\mathrm{z}=$ posição ao longo do tubo do reator recheado, ft. $\mathrm{u}=$ velocidade superficial = vazão volumétrica / área de secção transversal do tubo, $\mathrm{ft} / \mathrm{h}$. $\rho=$ massa específica do gás, $\mathrm{lb} / \mathrm{ft} 3 . \mathrm{G}=\rho \mathrm{u}=$ velocidade mássica superficial, $(\mathrm{g} / \mathrm{cm} 2 \mathrm{~s})$ ou (lbm/ft2h). A equação 12 é usada para calcular a queda de pressão na camada no capitulo 5 . 


\subsection{Modelo de radiação}

Em resumem os modelos de radiação usados pelo software ANSYS Fluent $\circledR$ se encontram na tabela 2:

Tabela 2. Descrição, vantagens e limitações dos Modelos de radiação.

\begin{tabular}{|c|c|c|}
\hline Modelo de radiação & Vantagens & Limitações \\
\hline $\begin{array}{l}\text {-Modelo de radiação de } \\
\text { transferência discreta } \\
\text { (DTRM): }\end{array}$ & Modelo simples & $\begin{array}{l}\text { Assume que todas as superfícies são difusivas, o que } \\
\text { significa que a reflexão de incidência a radiação da } \\
\text { superfície é isotrópica com respeito ao ângulo do } \\
\text { solido. } \\
\text { O efeito do espalhamento não é incluído. } \\
\text { A aplicação assume a radiação das cinzas. }\end{array}$ \\
\hline $\begin{array}{l}\text {-Modelo de radiação P- } \\
1 .\end{array}$ & $\begin{array}{l}\text { Pouca demanda do CPU } \\
\text { Incluo o modelo de espalhamento } \\
\text { Trabalha bem para efeitos da combustão. } \\
\text { Pode ser aplicado a geometrias complicadas }\end{array}$ & $\begin{array}{l}\text { Assume que todas as superfícies são difusivas. } \\
\text { O uso está restrito a radiação cinza ou não } \\
\text { O modelo P-1 Tende a exagerar a fluxos radiativos } \\
\text { de fontes de calor localizadas ou sumidouros. }\end{array}$ \\
\hline $\begin{array}{l}\text {-Modelo de radiação } \\
\text { Roseland }\end{array}$ & $\begin{array}{l}\text { Este modelo não resolve uma equação extra de } \\
\text { transporte } \\
\text { É mais rápido que o modelo P-1. } \\
\text { Exige menor recurso computacional }\end{array}$ & $\begin{array}{l}\text { è recomendado usar quando a espessura óptica não } \\
\text { excede de } 3 \text {. }\end{array}$ \\
\hline $\begin{array}{l}\text {-Modelo de radiação } \\
\text { Ordenada discreta (DO) }\end{array}$ & $\begin{array}{l}\text { Funciona com vários tipos de espessuras ópticas } \\
\text { è usado para superfícies de radiação que } \\
\text { participam da combustão. } \\
\text { O custo computacional é moderado }\end{array}$ & $\begin{array}{l}\text { O uso de este modelo está restrito a radiação cinza ou } \\
\text { não } \\
\text { Pode consumir muito recurso computacional }\end{array}$ \\
\hline $\begin{array}{l}\text {-Modelo de radiação } \\
\text { Superfície }- \text { superfície } \\
(\mathrm{S} 2 \mathrm{~S})\end{array}$ & $\begin{array}{l}\text { è bom para modelagens de transferência } \\
\text { radiativa. } \\
\text { Pode ser mais rápido que o modelo DTRM e o } \\
\text { modelo DO }\end{array}$ & $\begin{array}{l}\text { Não é sempre eficiente } \\
\text { Assume todas as superfícies difusivas } \\
\text { Assume radiação cinza } \\
\text { Não pode ser usado em problemas de condições } \\
\text { periódicas nem simetrias }\end{array}$ \\
\hline
\end{tabular}

O modelo de transferência de calor por radiação é importante em uma caldeira e é principalmente transportada por ondas desde a energia conteúda na chama até as paredes da fornalha e os feixes de tubos de reaquecimento.

\section{MARCO TEÓRICO}

Segundo Yin (2008), "a modelagem de caldeiras no leito fixo em caldeiras de biomassa envolve a simulação do solido ou combustível dentro da camada e ao mesmo tempo as reações da fase de gás dentro do forno de combustão. Usualmente softwares comerciais de CFD são utilizados pela engenharia, principalmente para resolver as equações de governo da fase de gás e a interação entre camada de combustível - gases, porém em termos de modelagem do leito fixo de combustão suas capacidades são muitas vezes limitadas. Três principais abordagens são consideradas na solução para este problema".

A primeira opção é um método simples, consiste em o uso das condições de entrada baseadas em medições experimentais sobre a superfície superior da camada de combustível, estas podem ser introduzidas através de uma função uniforme de posição sobre a superfície. Outras condições de entrada do modelo se calculam através do balanço de massa e calor dos componentes do combustível e a velocidade de ar primário.

O segundo método é o mais complexo e consiste em separar submodelos para calcular a temperatura, composição do gás e velocidade na superfície da camada. O código de CFD é usado para acoplar o submodelo do leito fixo e o fluxo radiativo emitido pela chama e pelas paredes de fornos até a superfície superior da camada de combustível e realimentada para a próxima iteração do modelo da camada. Além disso, devido à complexidade das reações no leito, há parâmetros desconhecidos e umas séries de simplificações têm que ser feitas, para justificar suposições duvidosas, se precisam realizar estudos de sensibilidade sobre os efeitos dos parâmetros-chave e a taxa de combustão (por exemplo, os efeitos do teor de umidade, densidade de partículas, tamanho, forma, poder calorífico, porosidade do leito, e os parâmetros de transferência de calor, capacidade de calor de gases, etc.).

O terceiro método é definir sub-rotinas User Definition Funtion (UDF) no código CFD. Este código contém os detalhes essenciais para caracterizar as interações da fase sólida com o gás. Uma forma simples de este método é supor 
a camada de biomassa como uma zona porosa e logo escrever uma função no modelo CFD descrevendo as propriedades como: a umidade, voláteis e a energia usada na secagem, desvolatilização e char da combustão. A abordagem da zona porosa é particularmente útil para situações em que as tendências globais do comportamento e o desempenho da caldeira precisam ser avaliados, em vez de onde os detalhes finos da combustão em leito têm que ser resolvidos.

A modelagem da combustão na camada não foi feita para este artigo devido a que para isso precisa-se maior desenvolvimento no tema, mas o calor fornecido pela combustão será modelado por uma fonte de calor.

Para a modelagem do escoamento na fase gasosa precisa-se selecionar o modelo de turbulência e radiação, para isso se realizo uma revisão bibliográfica analisando os modelos usados para cada caso especifico, na tabela 3 mostra-se esta descrição.

Tabela 3. Resumem das modelagens selecionadas pelos diferentes autores.

\begin{tabular}{|c|c|c|c|c|c|}
\hline Autor & Tipo de Caldeira & $\begin{array}{c}\text { Método usado para a } \\
\text { simulação da combustão }\end{array}$ & $\begin{array}{l}\text { Modelo de } \\
\text { turbulência }\end{array}$ & $\begin{array}{c}\text { Modelo de } \\
\text { radiação }\end{array}$ & $\begin{array}{l}\text { Transporte } \\
\text { de espécies }\end{array}$ \\
\hline Chaney, J. & $\begin{array}{l}\text { Pellet biomassa, caldeira de } 50 \\
\mathrm{~kW} \text {, grelha fixa. }\end{array}$ & 3 (código UDF) & $\mathrm{K}-\varepsilon$ & $P-1$ & Sim \\
\hline Sui, J. & $\begin{array}{l}\text { Briquetas de } 3 \text { tipos de } \\
\text { biomassa e carvão. Grelha fixa }\end{array}$ & 2 e 3 & $\begin{array}{l}\text { Standard } \\
\text { Realizable } \quad \mathrm{k}- \\
\text { épsilon }\end{array}$ & $\mathrm{P}-1$ & - \\
\hline Houshfar, E. & $\begin{array}{l}5 \text { tipos de biomassa, } 16 \mathrm{~kW} \text {, } \\
\text { grelha fixa }\end{array}$ & Experimento & - & - & - \\
\hline Beng, H. & Madeira, grelha fixa & 1 e 3 & $\mathrm{~K}-\varepsilon$ & - & Sim \\
\hline Miltner, M. & Caldeira 2MW, biomassa & 3 & SST-k- $\omega$ & DO & Sim \\
\hline Scharler, R. & $\begin{array}{l}\text { Caldeiras de } 0.5-10 \mathrm{MW} \text {. } \\
\text { Grelha fixa. }\end{array}$ & 1 e Eddy Dissipation Model & $\begin{array}{l}\text { Standard } \\
\text { Realizable } \quad \mathrm{k}- \\
\text { épsilon }\end{array}$ & DO & Sim \\
\hline Jurena. T. & Palha, Grelha fixa & 1 e 2 & - & - & Sim \\
\hline Yin, Ch. & Biomassa, Grelha fixa & 1 e 3 & - & - & Sim \\
\hline Yin, Ch. & $\begin{array}{l}108 \text { MW. Biomassa, Grelha } \\
\text { fixa }\end{array}$ & 1,2 e 3 & $\begin{array}{l}\text { k-épsilon } \\
\text { standard }\end{array}$ & DO & Sim \\
\hline Yang, Y. B. & $\begin{array}{l}\text { Biomassa. } \\
\text { movimento }\end{array}$ & 3 e 1 & $\begin{array}{l}\text { k-épsilon } \\
\text { standard }\end{array}$ & DO & Sim \\
\hline Porteiro, J. & $\begin{array}{l}\text { Pellet Biomassa. } 24 \quad \mathrm{~kW} \text {. } \\
\text { Grelha fixa. }\end{array}$ & 2 e 3 & $\begin{array}{l}\text { k-épsilon } \\
\text { standard }\end{array}$ & DO & Sim \\
\hline Collazo, J. & $\begin{array}{l}\text { Pellet Biomassa. } 24 \quad \mathrm{~kW} \text {. } \\
\text { Grelha fixa. }\end{array}$ & 1 e 3 & $\begin{array}{l}\text { k-épsilon } \\
\text { standard }\end{array}$ & DO & Sim \\
\hline
\end{tabular}

Os modelos de turbulência e radiação usados pelos autores para casos de estudo similares são: Modelo Standard Realizable k-épsilon como modelo de turbulência, este é usado para escoamentos turbulentos perto de corpos sólidos, mas além apresenta um comportamento aceitável para escoamentos longe das paredes do solido, e o modelo DO para radiação, em geral os modelos de radiação tem problemas para combustão, más segundo os autores o modelo DO apresenta más precisão que o modelo P-1.

\section{CASO DE ESTUDO}




\subsection{Caldeira do estudo}

A caldeira do estudo foi adquirida pela Universidade Federal de Itajubá e está instalada no laboratório do NEST, usa como combustível biomassa (lenha ou resíduos de madeira). Esta caldeira foi fabricada pela empresa AG-Therm, o desenho é mostrado na figura 3, e as caraterísticas do projeto mostram-se na tabela 4.
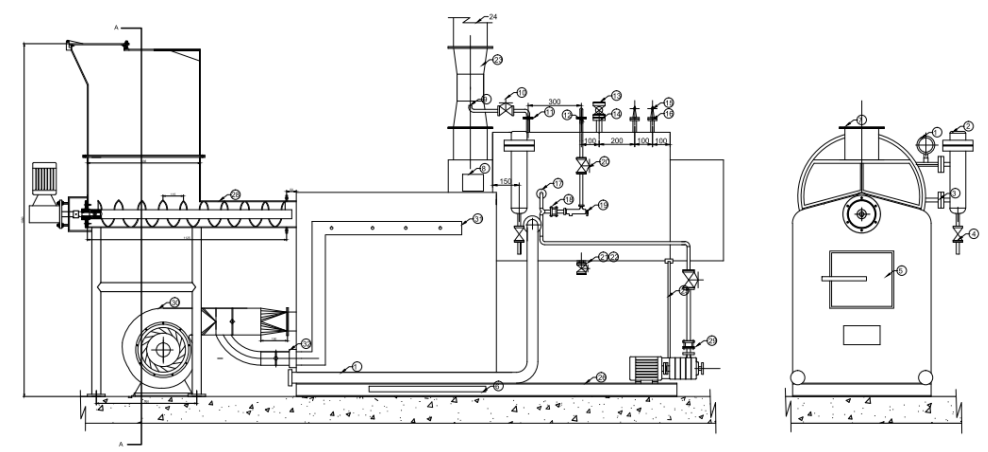

Figura 3. Esquema da caldeira piloto. Fornecido pela empresa AG-Therm

Tabela 4. Caraterísticas da caldeira piloto.

\begin{tabular}{|c|c|c|c|}
\hline \multicolumn{3}{|c|}{ Características } & Unidade \\
\hline \multicolumn{2}{|l|}{ Modelo } & Aquatubular & - \\
\hline \multicolumn{2}{|l|}{ Capacidade térmica } & $90(77386)$ & kWT (kcal/h) \\
\hline \multicolumn{2}{|c|}{ Pressão de vapor } & 10 & $\mathrm{kgf} / \mathrm{cm} 2$ \\
\hline \multicolumn{2}{|c|}{ Capacidade de produção de vapor } & 60 & $\mathrm{~kg} / \mathrm{h}$ \\
\hline \multicolumn{2}{|c|}{ Volume de gases gerados } & 90,50 & $\mathrm{Nm} 3 / \mathrm{h}$ \\
\hline \multicolumn{2}{|c|}{ Temperatura média dos gases } & 760 & ${ }^{\circ} \mathrm{C}$ \\
\hline \multicolumn{2}{|c|}{ Biomassa } & Carvão vegetal, resíduo de madeira, etc. & - \\
\hline \multicolumn{2}{|c|}{ Consumo de biomassa } & 37,70 & $\mathrm{~kg} / \mathrm{h}$ \\
\hline \multicolumn{2}{|c|}{ Umidade da biomassa considerada } & 30 & $\%$ \\
\hline \multicolumn{2}{|c|}{ Poder calorífico inferior } & 2700 & $\mathrm{kcal} / \mathrm{kg}$ \\
\hline \multirow{2}{*}{\multicolumn{2}{|c|}{ Área de suporte de queima }} & 0,20 & $\mathrm{~m} 2$ \\
\hline & & 400 & $\mathrm{~mm}$ \\
\hline \multirow[t]{2}{*}{ Dimensões aproximadas } & Comprimento & 500 & $\mathrm{~mm}$ \\
\hline & Altura & 1200 & $\mathrm{~mm}$ \\
\hline \multicolumn{2}{|l|}{ Tipo de grelha } & Plana tubular com refrigeração interna & \\
\hline
\end{tabular}

\subsection{Biomassa}

A biomassa que é modelada neste trabalho é madeira de eucalipto preparada, figura 4.

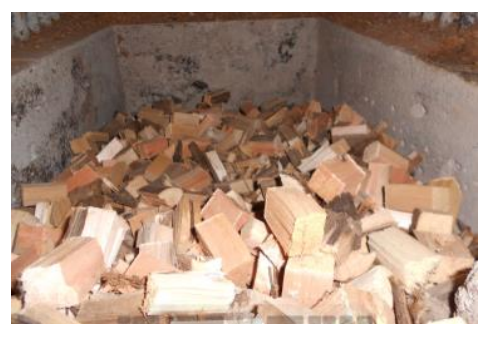

Figura 4. Madeira preparada na fornalha da caldeira

E a caraterização da biomassa foi feito num laboratório especializado os resultados são mostrados na tabela 5 .

Tabela 5. Caracterização da biomassa.

\begin{tabular}{|c|c|c|c|c|c|c|c|c|c|}
\hline \multicolumn{3}{|c|}{ Análise imediata } & \multicolumn{5}{|c|}{ Análise elementar } & PCI & PCS \\
\hline $\mathrm{FC}(\%)$ & $\mathrm{VM}(\%)$ & ASH (\%) & $\mathrm{C}(\%)$ & $\mathrm{H}(\%)$ & $\mathrm{O}(\%)$ & $\mathrm{N}(\%)$ & $\mathrm{S}(\%)$ & \multicolumn{2}{|c|}{$(\mathrm{MJ} / \mathrm{kg})$} \\
\hline 15,66 & 83,27 & 1,34 & 45,78 & 5,92 & 42,83 & 0,392 & 0,093 & 18.433 & 19.351 \\
\hline
\end{tabular}




\subsection{Testes}

Adicionalmente se realizaram 2 testes: o primeiro foi realizado para determinar as variáveis de entrada na modelagem como velocidade de entrada do ar primário e secundário, e medir a queda de pressão produzida pelo leito na fornalha. O segundo teste foi calcular algumas caraterísticas da camada como densidade e porosidade os cálculos se mostraram no capitulo 5 .

\section{AVANÇOS NA MODElagem da CALdeIRA PILOTO DE QUEIMA DE BIOMASSA EM LEITO FIXO}

Neste capitulo se mostraram os avanços da modelagem da combustão na caldeira piloto. Em primeiro lugar se mediu o volume interno da fornalha (geometria do modelo) e alguns valores de entrada como: a velocidade do ar e a pressão; também se cálculo as condições de contorno, por exemplo, material nas paredes da fornalha. Posteriormente, se elaboro a geometria do modelo, considerando algumas simplificações que não afetam o processo de combustão, mas reduzem o consumo computacional na solução dos resultados. Consequentemente se projeto a malha, este processo é muito importante para lograr precisão nos resultados. Finalmente os avanços da modelagem se dividem em duas etapas consequentes, que são: na primeira modelagem se caracteriza o leito poroso que representa a camada de biomassa, e na segunda modelagem se simulo a transferência de calor na fornalha, incluindo o modelo de transferência de calor por radiação, devido a uma fonte térmica equivalente a energia entregada pela biomassa na combustão.

Estas simulações foram realizadas em um laptop de marca ASUS®, com 8 Gb de memória RAM. O software usado é ANSYS Fluent® V.14.

\subsection{Projeto da Geometria}

A geometria do modelo foi realizada em o software comercial Inventor Autodesk $®$. Figura 5.
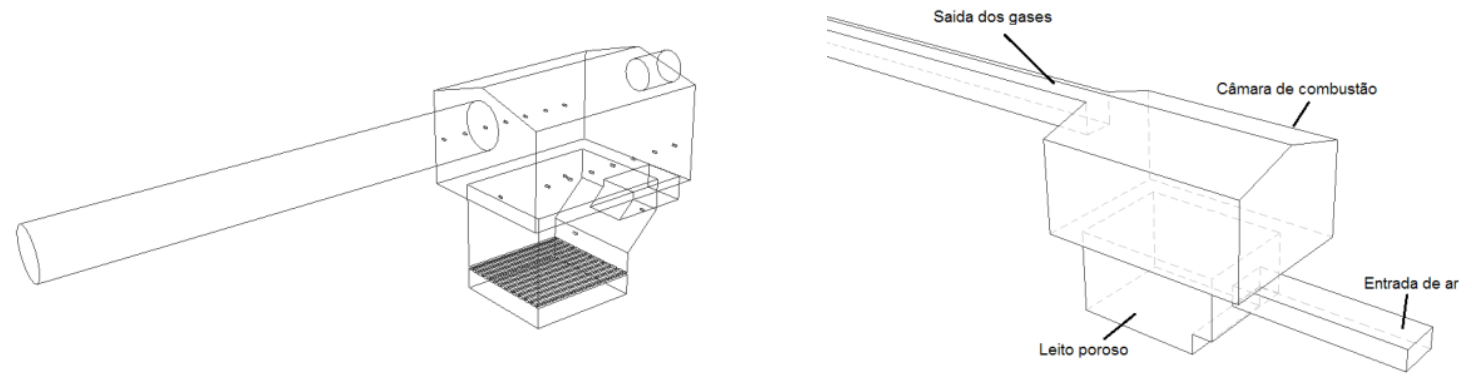

Figura 5. Esquema da geometria da caldeira piloto para a modelagem em CFD.

\subsection{Cálculos de entrada da modelagem}

As variáveis de entrada foram medidas com equipamentos especializados na caldeira em operação, na tabela 6 amostram-se as variáveis de contorno de entrada para a modelagem.

Tabela 6. Variáveis de contorno de entrada na modelagem.

\begin{tabular}{|l|c|}
\hline \multicolumn{1}{|c|}{ Variável de Entrada } & Valor do Teste \\
\hline Vazão de ar primário $\left(\mathrm{m}^{\wedge} 3 / \mathrm{h}\right)$ & 480 \\
\hline Vazão de ar secundário $\left(\mathrm{m}^{\wedge} 3 / \mathrm{h}\right)$ & 5 \\
\hline Velocidade de ar primário $(\mathrm{m} / \mathrm{s})$ & 0.5 \\
\hline Velocidade de ar secundário $(\mathrm{m} / \mathrm{s})$ & atm \\
\hline Pressão de saída & 180 \\
\hline Pressão antes da camada $(\mathrm{pa})$ & 36 \\
\hline Pressão depois da camada $(\mathrm{pa})$ & 330 \\
\hline Temperatura nas paredes $(\mathrm{K})$ & $51-37,7$ \\
\hline Vazão de biomassa $(\mathrm{kg} / \mathrm{h})$ & 266,3 \\
\hline Densidade da biomassa na camada $\left(\mathrm{kg} / \mathrm{m}^{\wedge} 3\right)$ & \\
\hline
\end{tabular}




\begin{tabular}{|l|c|}
\hline Poder calorifico (madeira eucalipto) (MJ/kg) & 18433 \\
\hline Número de Reynolds & 6102,2 \\
\hline Modelo de turbulência & $\mathrm{k}-\varepsilon$ Realizable \\
\hline Escoamento & $\mathrm{Ar}$ \\
\hline Material da biomassa & Madeira \\
\hline
\end{tabular}

\subsection{Projeção da malha}

O primeiro passo é fazer o cálculo aproximado do cumprimento de cada célula na malha e definir a estratégia na projeção da malha.

Para o cálculo do cumprimento de cada célula y é necessário conhecer um valor aproximado da variável adimensional $\mathrm{y}^{+}$, a relação do y e o $\mathrm{y}^{+}$é diretamente proporcional e um valor de y pequena garantia resultados exatos, para escoamentos turbulentos um valor aceitável do $\mathrm{y}^{+}$está entre 0 e 120 , para este caso do estudo o y ${ }^{+}$será de 80 para reduzir o consumo computacional sem perder a precisão nos resultados. Os resultados do cálculo do y mostram-se na tabela 7.

Tabela 7. Cálculo aproximado do cumprimento das células da malha.

\begin{tabular}{|c|c|c|c|}
\hline $\mathbf{R e}$ & $\mathbf{U}(\mathbf{m} / \mathbf{s})$ & $\mathbf{y +}$ & $\mathbf{y}(\mathbf{c m})$ \\
\hline 6102,12 & 1,5 & 80 & 7 \\
\hline
\end{tabular}

A estratégia para a projeção da malha é fazer uma malha uniforme na fornalha toda, devido que a turbulência produzida pela queima da biomassa é complexa sendo difícil reconhecer zonas de refinamento. Então a malha é uniforme com cumprimento entre nodos de $7 \mathrm{~cm}$, figura 6.

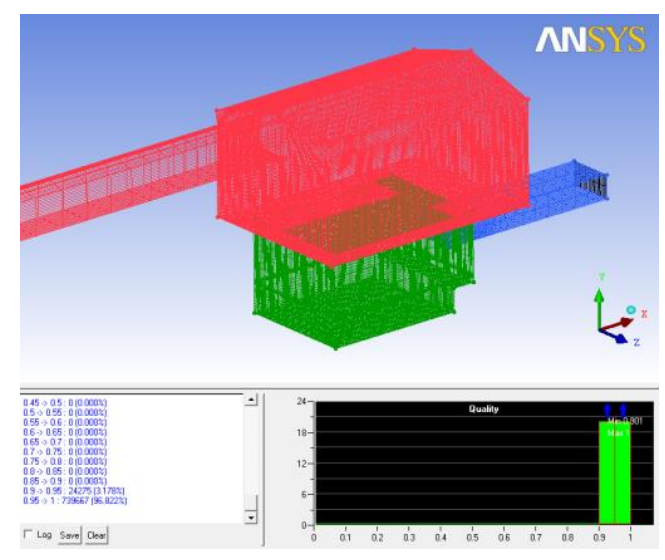

Figura 6. Projeção da malha.

$\mathrm{Na}$ anterior figura pode se ver a qualidade da malha segundo o software ICEM CFD $®$, a qualidade se qualifica de 0 a 1 , os elementos para modelagem encontram se entre 0.9 e 1.0 sendo uma malha adequada.

\subsection{Modelagem do meio poroso.}

A caracterização do meio poroso foi realizada pelo teste onde foi medido a queda de pressão através do leito e usando as equações do capitulo 2. Os resultados são mostrados na tabela 8.

\begin{tabular}{|l|c|c|c|}
\hline & Madeira & Ar & Total \\
\hline Volume $\left(\mathrm{m}^{\wedge} 3\right)$ & 0,0672 & 0,0678 & 0,1350 \\
\hline Massa $(\mathrm{kg})$ & 35,9512 & 0,0142 & 35,9654 \\
\hline Porosidade & \multicolumn{3}{|c|}{0,00014} \\
\hline Permeabilidade $\alpha\left(1 / \mathrm{m}^{\wedge} 2\right)$ & \multicolumn{3}{|c|}{28,93} \\
\hline Coeficiente de perdas inerciais $(1 / \mathrm{m})$ & \multicolumn{3}{|c|}{2,5} \\
\hline Diâmetro caraterístico $(\mathrm{cm})$ & \multicolumn{3}{|c|}{} \\
\hline Velocidade $(\mathrm{m} / \mathrm{s})$ & \multicolumn{3}{|c|}{} \\
\hline
\end{tabular}


Com a equação de Egun (capitulo 2) se cálculo a queda de pressão no leito V.S. a velocidade do escoamento, na figura 7 mostra se a relação da queda de pressão com a velocidade no leito comparada com outra gráfica em uma caldeira industrial.
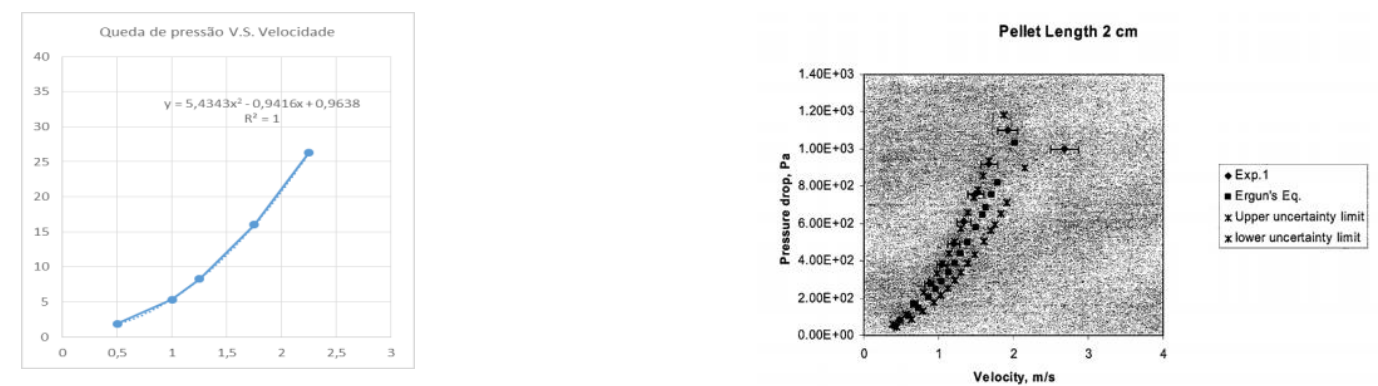

Figura 7. Queda de pressão V.S. Velocidade através do leito fixo.

\subsection{Modelagem com fonte térmica.}

O resultado do perfil de velocidades e de temperaturas é mostrado na figura 8.
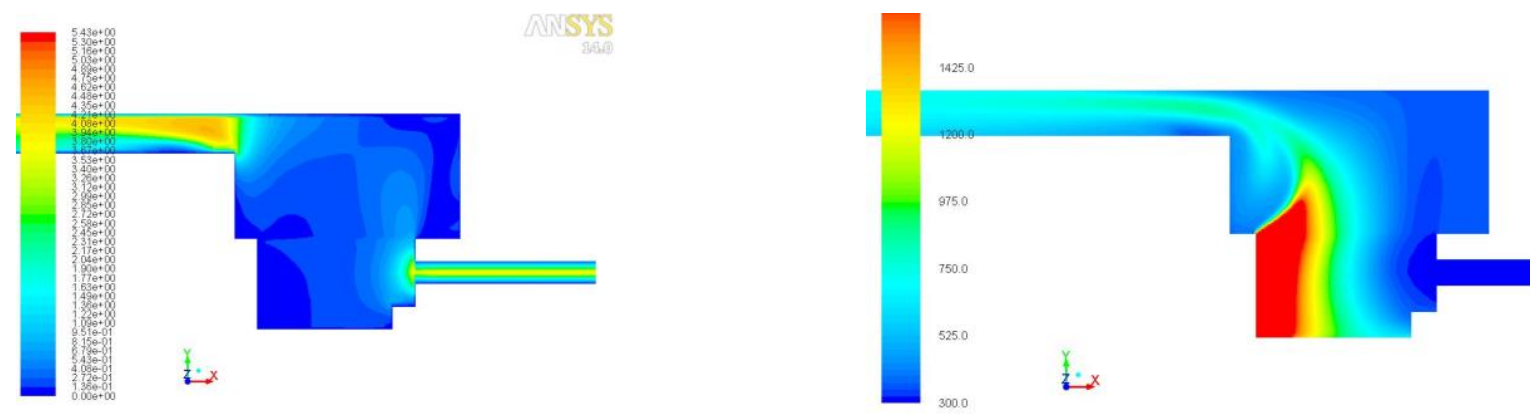

Figura 8. Perfil de velocidades e de temperaturas na fornalha

O perfil de velocidades dentro da fornalha mostra um comportamento similar ao comportamento do escoamento do teste onde a maioria do ar sai do leito fixo pela parte direita da camada próximo a entrada de ar primário devido a resistência no leito na direção X. O cálculo da temperatura próxima dos feixes de tubos de troca de calor da caldeira está entre 700 e $900 \mathrm{~K}$ similar ao valor de temperatura dos gases fornecido pelos dados de projeto na caldeira.

Para visualizar o comportamento do escoamento dentro do leito foram feitas dos líneas de cálculo dentro da camada onde como se mostra na figura 9 (esquerda). A figura 9 (direita) mostram a queda de pressão e temperatura através do leito.
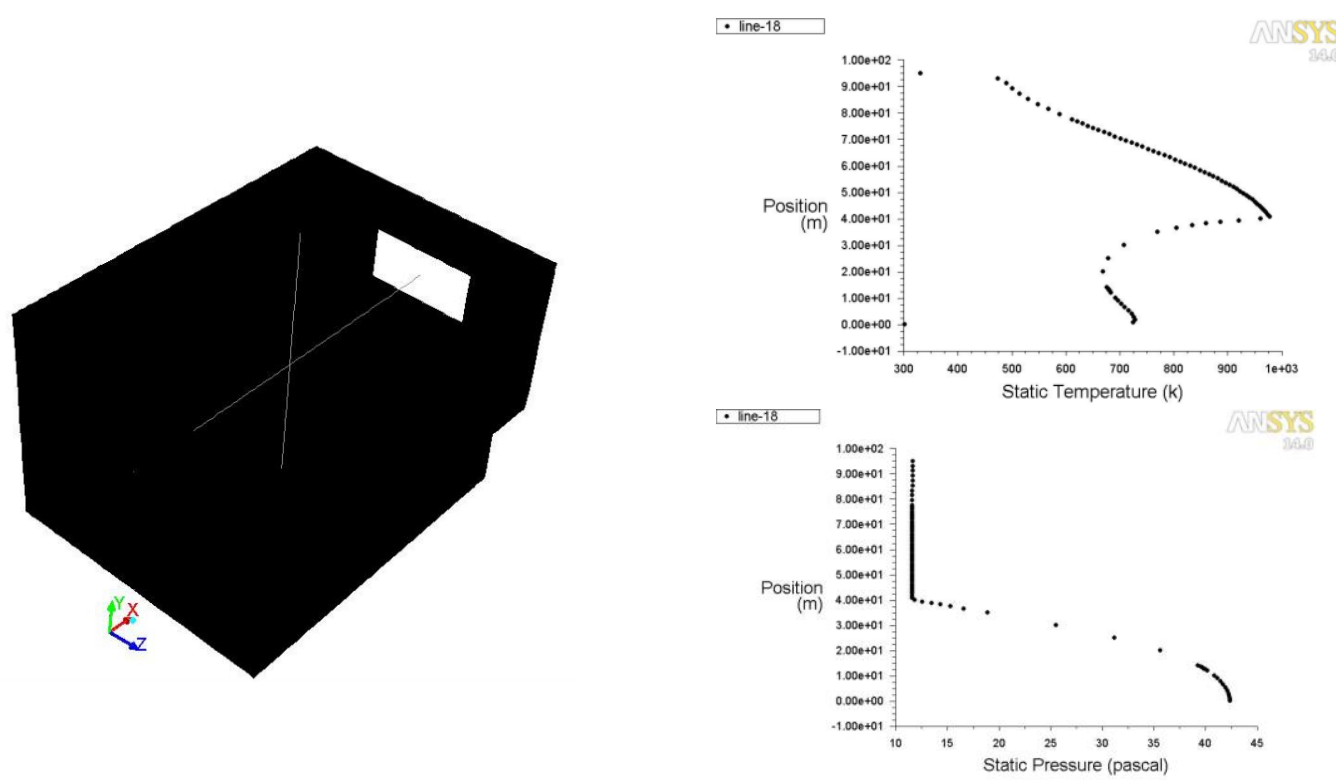

Figura 9. Temperatura e pressão através da camada na fornalha. 
Os resultados mostram um aumento na temperatura justo depois da camada até $1100 \mathrm{~K}$ aproximadamente o qual trem relação com o lido na literatura e a pressão presenta o comportamento adequado comparado com os resultados dos testes.

\section{REFERÊNCIAS}

Azevedo, J. L. F. 1999. Mecânica dos fluidos computacional. São José dos Campos: Embraer.

Global energy assessment (GEA). 2012. Toward a Sustainable Future Key Findings Summary for Policy makers Technical Summary. Summary Rio + 20. USA, New York.

Bazzo, E. 1995. Geração de Vapor. Universidade Federal de Santa Catarina. 2. Ed. Florianópolis. Brasil.

Chungen Yin, L. R. 2008. Mathematical Modelling and Experiemntal Study of Biomass Combustion in a Thermal 108 MW Grate Fired Boiler. Energy \& Fuels, 22, 1380-1390.

Chaney, J. Liu, H. Li, J. 2012. An overview of CFD modelling of small-scale fixed-bed biomass pellet boilerswith preliminary results from a simplified approach. Energy Conversion and Management. 63. 149-156.

Sui, J. Xu, X. Zhang, Bo. Huang, Ch. Lv, J. 2013. A Mathematical Model of Biomass Briquette Fuel Combustion. Energy and Power Engineering, 5, 1-5.

Beng, H., B. 2008. Breeding a better stove: The use of computational fluid Dynamics and Gentic Algorithms to optimise a wood burning stove for Eritrea. The University of Nothtingham School of Mechanical, Materials and Manufacturing engineering. Thesis submitted to the University of Nottingham for the degree of Doctor of Philosophy.

Scharler. R., Obernberger. I. Deriving guidelines for the design of biomass grate furnaces with CFD analysis - a new Multifuel-Low-Nox furnaces as example. Institute of Chemical Engineering Fundamentals and Plant Engineering. Graz University of Tecnology. Bioenerg. Graz. Austria

Jurena, T. 2011. Energy Considerations in CFD Modelling of Biomass Combustion in an experimental Fixed-Bed Reactor. Chimical Engineering Transactions. Vol 35.

Yin, Ch. Rosendahl, L., A. Kaer, S., K. 2008. Grate-firing of biomass for heat and power production. Progress in Energy and Combustion Science, 34. 725-754.

Yin Ch. Rosendahl, L., A. Kaer, S., K. Clausen, S. Hvid, S., L., e Hille, T. 2008. Mathematical Modeling and Experimental Study of Biomass Combustion in a Thermal 108 MW Grate-Fired Boiler. Institute of Energy Technology, Aalborg University. Energy \& Fuels, 22, 1380-1390.

Yang, Y. B., Ryu. C., Goodfellow. J., Nasserzadeh. V. e Swithenbank. J. 2004. Modelling Waste Combustion in grate Furnaces. The University of Sheffield. UK. Process Safety and Enviroment Protection, 82 (B3): 208-22.

Porteiro, J., Collazo. J., Patiño. D., Granada. E., Gonzales. J. C. e Míguez. J. L. 2009. Numerical Modeling of a Biomass Pellet Domestic Boiler. UniVersity of Vigo, Thermal Engines. Energy \& Fuels, 23, 1067-1075.

Collazo. J., Porteiro, J., Míguez. J. L. e Gómez. M. A. 2012. Numerical simulation of a small-scale biomass boiler. UniVersity of Vigo, Thermal Engines. Energy Conversion and Management 64 87-96.

Fogler. H. S. 1999. Elementos de Enghenaria das reações químicas. Terceira edição. University de Michigan.

Barros, R. W. 2005. Avaliação Teórica e Experimental do Motor Stirling Solo 161 Operando com Diferentes Combustíveis. Itajubá, Dissertação (Mestrado em Conversão de Energia) - Instituto de Engenharia Mecânica, Universidade Federal de Itajubá. 131p.

Noguera, A. L. 2012. Análise dos efeitos das condições operacionais de um gaseificador co-corrente de duplo estágio na qualidade do gás. Dissertação de Mestrado. Engenharia de Energia. Universidade Federal de Itajubá. Itjúba. Brasil.

Majumdar, P. 2011. Computational Fluid Dynamics Analysis of Turbulent Flow. Department of Mechanical Engineering, Northern Illinois University, Illinois. USA. P. 255-291.2011.

Development of generalised model for grate combustion of biomass. Final Report. Institute of Energy Technolog y Section for Fluid mechanics and Combustion Technology. Aalborg University. 2007. 


\author{
FOR THE PAPERS WRITTEN IN PORTUGUESE OR SPANISH, IT IS \\ NECESSARY TO INCLUDE THE ABSTRACT IN ENGLISH (TIMES NEW \\ ROMAN, BOLD, SIZE 14, center) \\ (single blank line size 14) \\ First Author's Name, e-mail ${ }^{1}$ (Times New Roman, Bold, size 10, center) \\ Second Author's Name, e-mail ${ }^{2}$ (Times New Roman, Bold, size 10, center) \\ Third Author's Name, e-mail ${ }^{3}$ (Times New Roman, Bold, size 10, center) \\ (single space line, size 10) \\ ${ }^{1}$ Institution and address for first author (Times New Roman, size 10, center) \\ ${ }^{2}$ Institution and address for second author (Times New Roman, size 10, center) \\ ${ }^{3}$ Institution and address for third author (Times New Roman, size 10, center) \\ Same format for other authors and institutions, if any (double space line, size 10, center)
}

Abstract. The purpose of these instructions is to serve as a guide for formatting papers to be published in the Proceedings of the CNMAI 2014. The abstract should describe the objectives, the methodology and the main conclusions of the paper in less than 300 words in a single paragraph. It should not contain either formulae or bibliographic references. (Times New Roman, italic, size 10)

(single space line, size 10)

Keywords: keyword 1, keyword 2, keyword 3 ... (up to 5 keywords) (Times New Roman, italic, size 10) 